\title{
Wireless Sensor Network based Accident Avoidance Architecture for the Fleet of Long Route vehicles
}

Ms. Jyoti.R.Dubey ${ }^{1}$, Dr. Ankit R. Bhavsar ${ }^{2}$, Dr. Harshal A. Arolkar ${ }^{3}$

\author{
${ }^{1}$ Assistant Professor FCAIT, GLS University \\ ${ }^{2}$ Assistant Professor FCAIT, GLS University \\ ${ }^{3}$ Associate Professor, FCAIT, GLS University, Ahmedabad, Gujarat, India

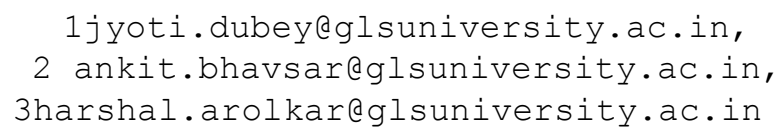

Abstract.Transportation is basic service required by all citizens. Long route transportation is norm today for transporting goods and persons. Road transport has remained one of the important means of transportation. Increase in population and motorization in the country along with expansion of road network contributes to the number of road accidents, injuries and mortalities, as well as loss of productivity. Safety of the passengers, drivers and property is very important issue when we talk about long route travelling. There are many issues that endanger the safety of people and vehicle during long route transportation. Some of them are driver's negligence while driving, overtaking by the vehicles, over speeding of vehicles on the highways, health and mental state of the driver while driving, mechanical issues of the vehicle, sudden arrival of animals on highways, unfamiliar road conditions, negotiating with difficult winding roads, road traffic, overloading of the vehicles and many more. To ensure safety of vehicle and people it is very important to be more cautious and take proper safety measures while driving for long routes. In this research paper we have proposed wireless sensor network based architecture that monitors various parameters that needs to be considered effectively for avoidance of accidents on long routes.

Keywords: WSN, Sensor, Transceiver, ADC, Motes

Received on 06 September 2017, accepted on 13 November 2017, published on 28 December 2017

Copyright († 2017 Jyoti.R.Dubey et al., licensed to EAI. This is an open access article distributed under the terms of the Creative Commons Attribution licence (http://creativecommons.org/licenses/by/3.0/), which permits unlimited use, distribution and reproduction in any medium so long as the original work is properly cited.

doi: 10.4108/eai.28-12-2017.153521

\section{Wireless Sensor Network (WSN)}

A Wireless Sensor Network is one kind of network that includes a large number of mobile, self-directed, low powered sensor nodes called motes. These networks consists of a huge number of spatially distributed, little, battery-operated, embedded devices. These devices are networked to caringly collect, process, and transfer data to the operators. The nodes are tiny computers, which work jointly to form the networks. ${ }^{[16]}$ 
A Wireless Sensor Network (WSN) includes hundreds of large number of low power multifunctional sensor nodes, operating within the unattended environment, and having sensing, computation and communication capabilities. The essential parts of a node undoubtedly are a sensor unit, an ADC (Analog to Digital Converter), a CPU, an electrical unit as well as a communication unit. ${ }^{[17]}$

WSN can be used in variety of fields which may include military, healthcare, environmental, biological, home and other commercial applications. ${ }^{[16]}$

A collection of sensor nodes collect the data from the surroundings to achieve specific application objectives.Sensor nodes are used for constant sensing, event detection and local control of actuators. ${ }^{[16]}$

\section{$2 \quad$ Accident Scenario in India}

Road safety is a matter of serious concern, its magnitude, gravity and the kind of negative impact it puts on economy, public health and welfare of people is catastrophic. Road accidents are one of the foremost causes of injuries, deaths, disabilities and loss of properties.

As per the annual report on "Road accidents India-2015" by JPRI (JP Research India Pvt Ltd.) the highest accident severity, that is road accidents deaths per 100 accidents is highest in Mizoram that is 102.9. The state wise severity of accidents is being represented in the Fig. 1. ${ }^{[18]}$

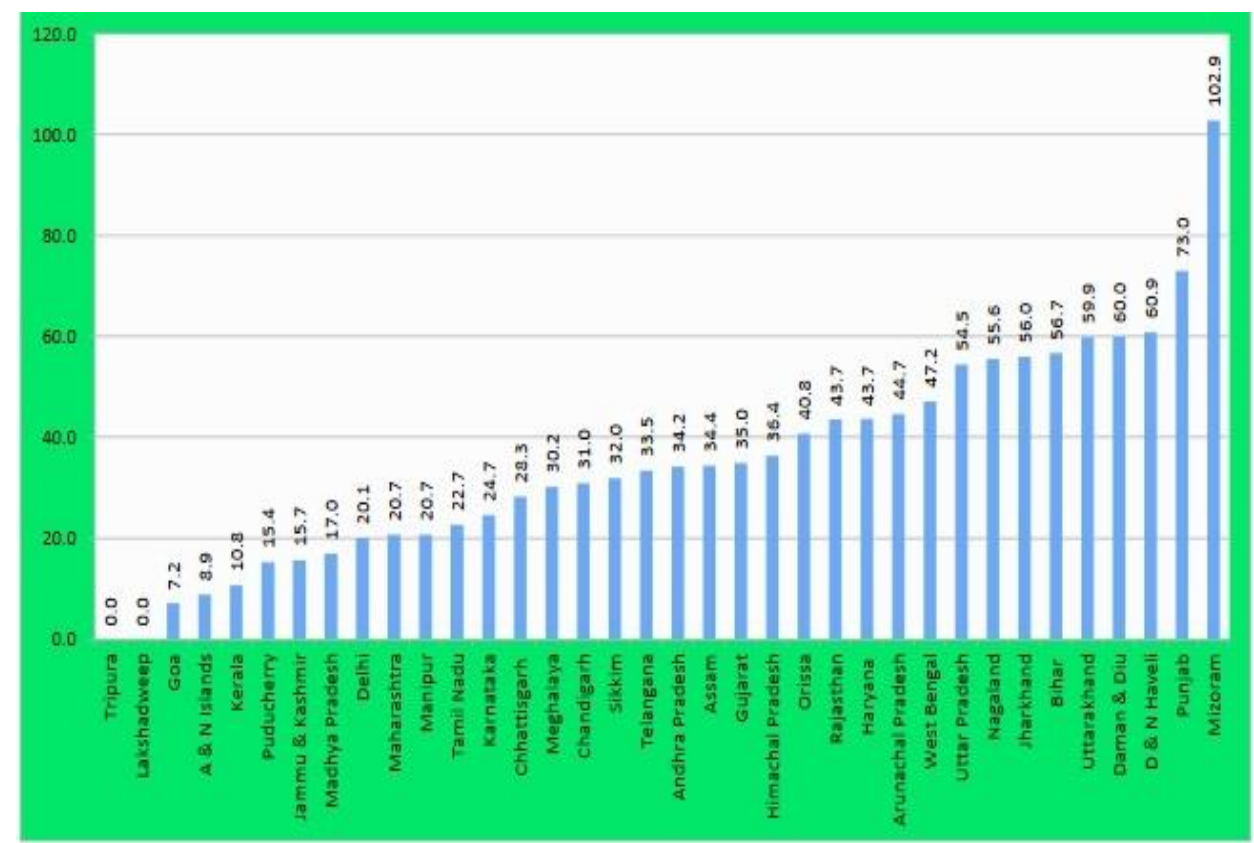

Fig. 1.Severities of road accidents across States during 2015 ${ }^{[18]}$ 
Fig. 2.is the comparison of the percentage shares of road accidents across the 13 states as per year (2014-15). Tamil Nadu has highest percentage of road accidents across the 13 major states of India. ${ }^{[18]}$

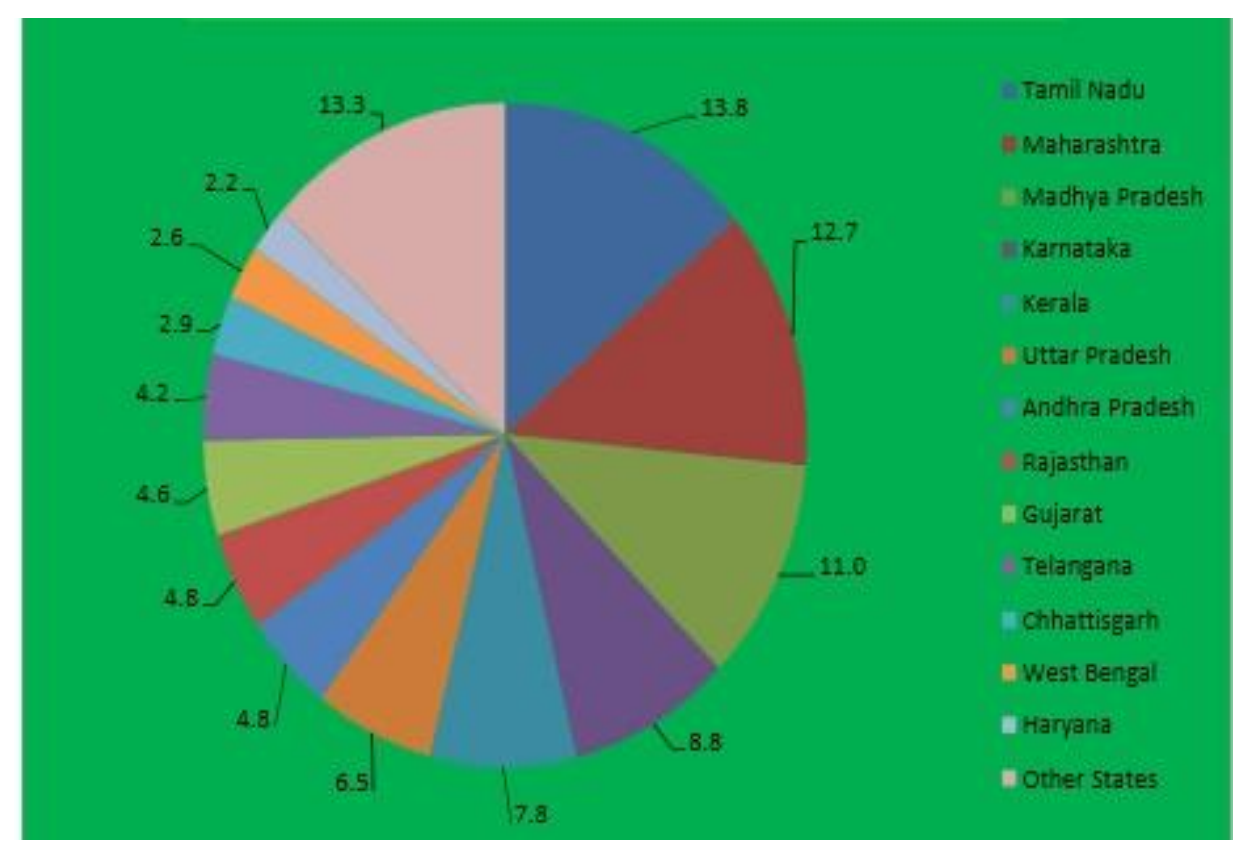

Fig. 2.Percentage number of road accidents in year $2015^{[18]}$

JPRI (JP Research India Pvt Ltd.) has analyzed 88 fatal or serious accidental cases. On the basis of this analysis JPRI has found the factors contributing to fatal or serious accidents. Various influensive factors are abnormal driver behavior, poor vehicle maintenance and road infrastructure. From Table 1. it was found that poor vehicle maintenance has highest influence of all, it contributes (49\%), followed by combination of driver behavior (Human) and infrastructure i.e. (35\%), while infrastructure related problems are only $6 \% \cdot{ }^{[18]}$ Fig. 3 is representing the graphical view of Table 1.

Table 1.Figure. 3 Influence of each factor in the occurrence of accidents ${ }^{[18]}$

\begin{tabular}{|l|c|c|}
\hline \multicolumn{1}{|c|}{ Factor } & Alone & All Combinations \\
\hline Driver Behavior (Human) & $49 \%$ & $91 \%$ \\
\hline Poor Vehicle Maintenance (Vehicle) & $3 \%$ & $10 \%$ \\
\hline Abnormal Road Condition (Infrastructure) & $6 \%$ & $44 \%$ \\
\hline
\end{tabular}




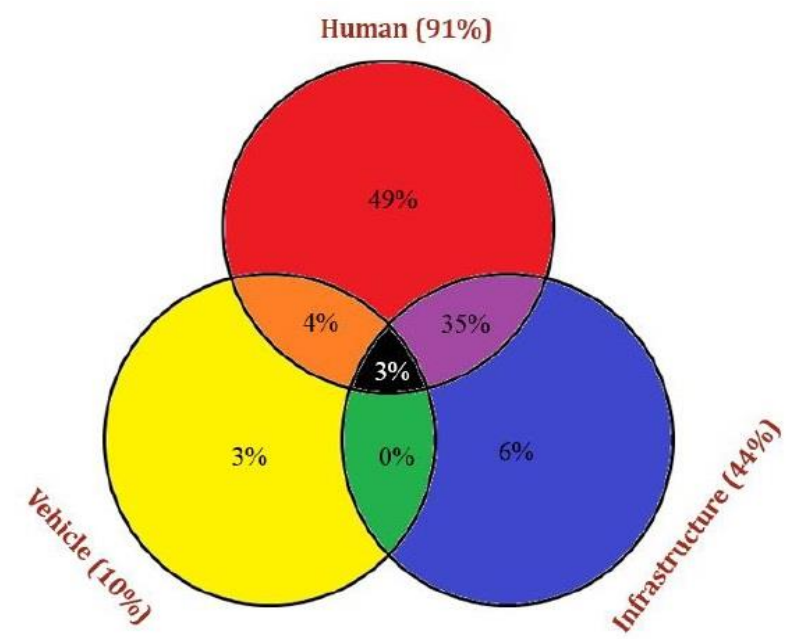

Fig. 3. Distribution of 211 Accidents by Contributing Factors influencing the Occurrence of Accidents

\section{Related Work}

WSN is being used in monitoring applications such as health monitoring, driver behavior monitoring in order to ensure safety and security. [6], [7], [8], [9], [10], [11], [12], [13], [14], [15], [16], [17].

A. Mounika et.al.[1] has proposed a non-intrusive bio-potential measurement system for driver's health monitoring and fatigue detection. The entire system composed of ARM7TDMI microcontroller, LCD display, alcohol sensor, eye blink sensor, ECG sensors, GPS system, GSM, Motor drive. The researcher proposes bio- potential measurement system that will cause less mental and physical load to the drivers. The system proposes how to measure bio-potential signals without the requirement of electrodes which otherwise needs to be in contact with human body which in turn causes hindrance to driver's while driving. The eye blink sensor continuously measures blinking of eye and sends the data to a microcontroller. GSM modem is used to trace the vehicle and also to inform to control room. ECG sensors generate electrical potentials on the basis of muscle cell contraction and relaxation. In case the pulse width of the driver is high then the vehicle is stopped and position of the vehicle is traced by GPS system and intimation is given instantly to the concerned doctor to reach the location of the vehicle to provide medical help to the driver.

Adnan K. Shaout et.al.[2] has proposed a practical and economical way to capture, measure, and alert driver of inefficient and unsafe driving. The proposed solution consists of a mobile application, running on a modern smart phone device, paired with a compatible OBD-II (On-board diagnostics II) reader, An OBD-II reader is a device used for troubleshooting problems with a vehicle or retrieve real time performance data by connecting directly to a vehicle's ECU (Engine Control Unit). The application 
proposed by the researcher is a prototype it's not implemented yet. The research is focusing on detecting mechanical issues with the vehicle rather than identifying or monitoring driver's behavior and environment. However this research could be extended to merge with monitoring driver's behavioral parameters while driving in real time.

D. Sowmya et.al.[3] has proposed a vital system for detecting and monitoring driver's behavior on the basis of information obtained from various sensors to ensure road safety by alerting the driver and other vehicles on the road in case of abnormal driver behavior. The behaviour here can be classified as normal behavior, drunk behavior, fatigue and reckless behavior. The researcher were able to capture various static and dynamic aspects of behavior which helped to understand decision making capability of the driver in different situations.

Joel C.McCall et.al.[4] has proposed Driver Attention Monitoring System. Aggregated outcomes from LISA-Q test bed that capture video, audio and vehicle information, Laser Radar information and information gathered from GPS system were considered as input to the system for evaluating driver's behavioral state. This system involves capturing and synchronizing data from many different types of sensors and generating relevant metrics from this information. By combining a wide variety of different sensors and metrics, a robust estimate of the driver's physical and mental state is created.

Luis M. Bergasa et.al.[5] has proposed a non-intrusive prototype computer vision system for determining driver's level of vigilance. The prototype has focused on calculating six parameters like PERCLOS (percentage of eye closure), eye closure duration, blink frequency, nodding frequency, face position and fixed gaze. The architecture of the proposed system consists of modules such as image acquisition, pupil detection and tracking, visual behaviors and driver vigilance. Data obtained by evaluating all the parameters is fed into a fuzzy classifier to evaluate the level of driver's inattentiveness. Alarm is activated to intimate the driver if level of inattentiveness is found above a predetermined threshold.

\section{Proposed architecture for accident avoidance}

In this research paper we have proposed a wireless sensor network based architecture that focuses on accident avoidance for vehicles that usually travel on long routes. Our proposed architecture focuses on monitoring driver's level of distraction by monitoring visual parameters, health condition, driving pattern of the driver. It also monitors road and traffic conditions, sudden arrival of animal on the highways. 

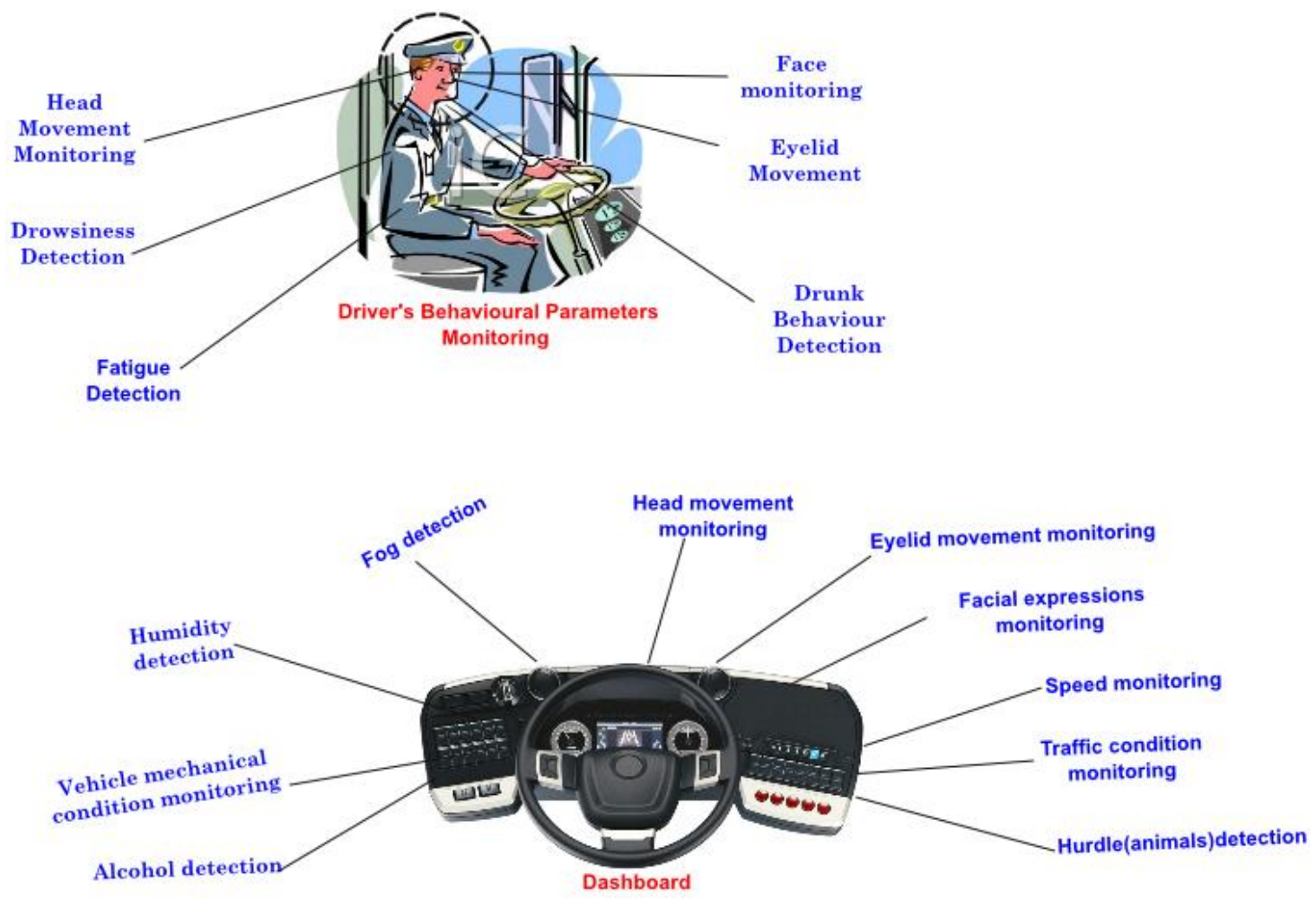

Fig. 4 Pictorial representation of the driver behavior and dashboard of the vehicle

Further we intend to observe over speeding of the vehicles. A nonintrusive system capable of monitoring the said parameters will be deployed. In case of any abnormal occurrence of events, the designated sensor will sense the event and informs its occurrence to the nearest toll plaza (Control room). The possible controlling actions like setting of an alarm within a vehicle, controlling speed of vehicle, intimating nearby vehicles etc can then be taken.

Fig.4. represents various parameters of driver that could be monitored by the proposed accident avoidance architecture. The parameters monitored here are, head movement, face monitoring, eyelid movement, drunken behavior detection, drowsiness detection and fatigue detection.

The dashboard seen in Fig.4 represents different tasks that are to be performed by various sensors or components fitted on it. The said sensors will be used to monitor mechanical condition of the vehicle, fog, humidity, driver's eyelid movement, driver's head movement, speed of vehicle and alcohol consumption by driver. 


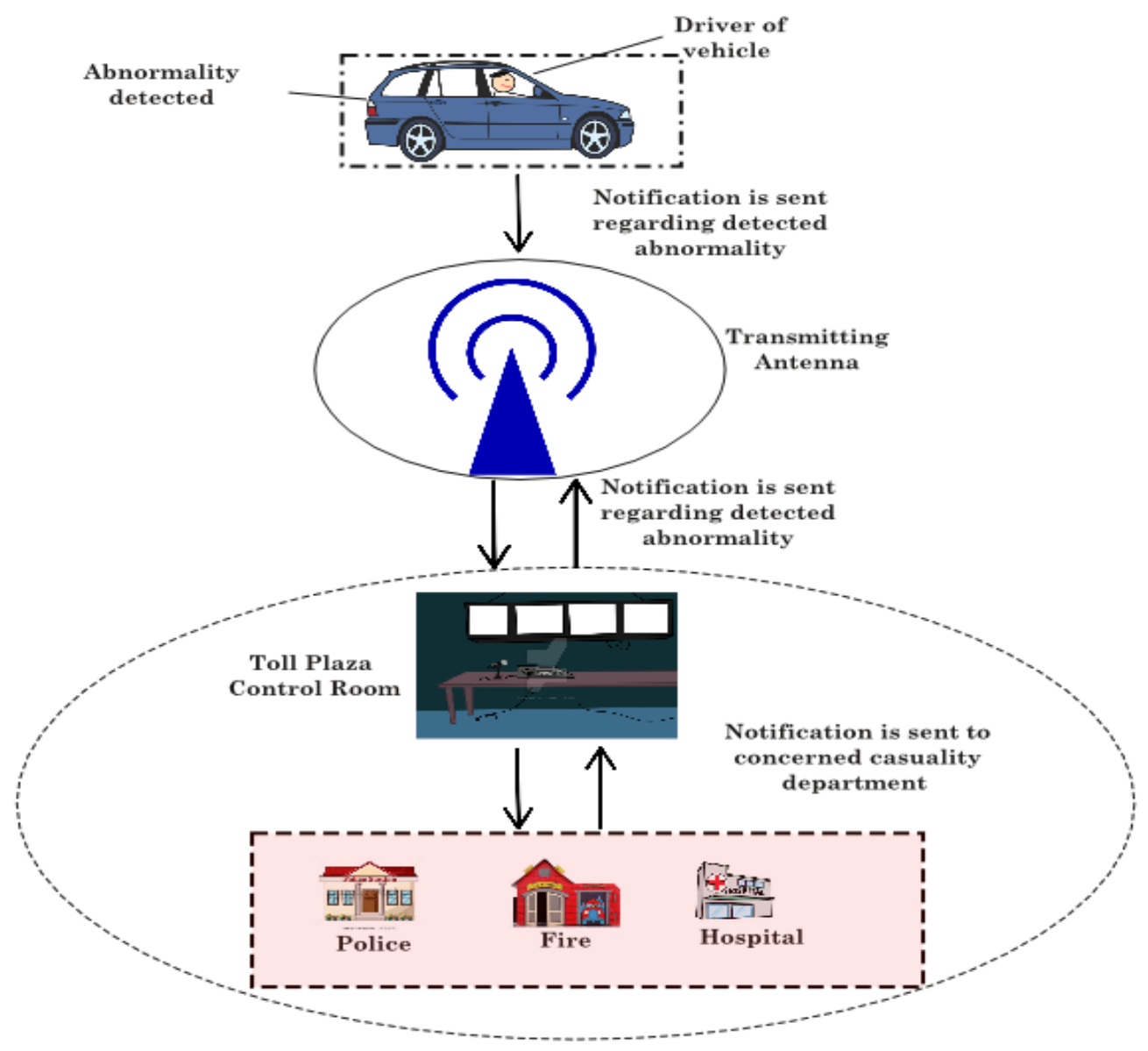

Fig. 5. Basic communication architecture for accident avoidance

\subsection{Basic architectural flow}

Fig.5. shows basic communication flow of our architecture. The communication flow in this architecture is based on three entities mainly vehicle, transmission media and control system. Vehicles that need to be monitored must be equipped with the smart system, which is capable of transmitting the required information pertaining to vehicle and driver's behavior. Antenna acts as a junction for transmitting required information. Toll plaza/control room is a place from where the corresponding actions can be initiated after receiving and analyzing the information obtained from vehicle.

\subsection{Detailed architecture}

Fig.6. represents the elaborated view of the architecture proposed by us. As per the proposed architecture the vehicles in consideration must be equipped with smart sys- 
tem. Architecture has been divided into three main zones. The three zones can be categorized as: Activity zone, Notification zone and Control zone.

Activity zone: It is the area where the abnormality would be detected either in driver behavior or vehicle system. The components of the activity zone can be vehicle in which abnormality is being detected, cluster antenna and surrounding/neighbouring vehicles. Every antenna has probable range of $15 \mathrm{~km}$ which will create a virtual cell to send and receive any data within a diameter of $15 \mathrm{~km}$ around it. The vehicle equipped with smart system which is passing through the cell can transmit driver behavior or vehicle system data to the corresponding cell cluster antenna and to the nearby vehicle which are at the probable distance of $1 \mathrm{~km}$ from it. Provided both vehicles should be in the same virtual cell of antenna as mentioned above.

Activity zone activities can be categorized as: Probable accident condition and Actual accident condition.

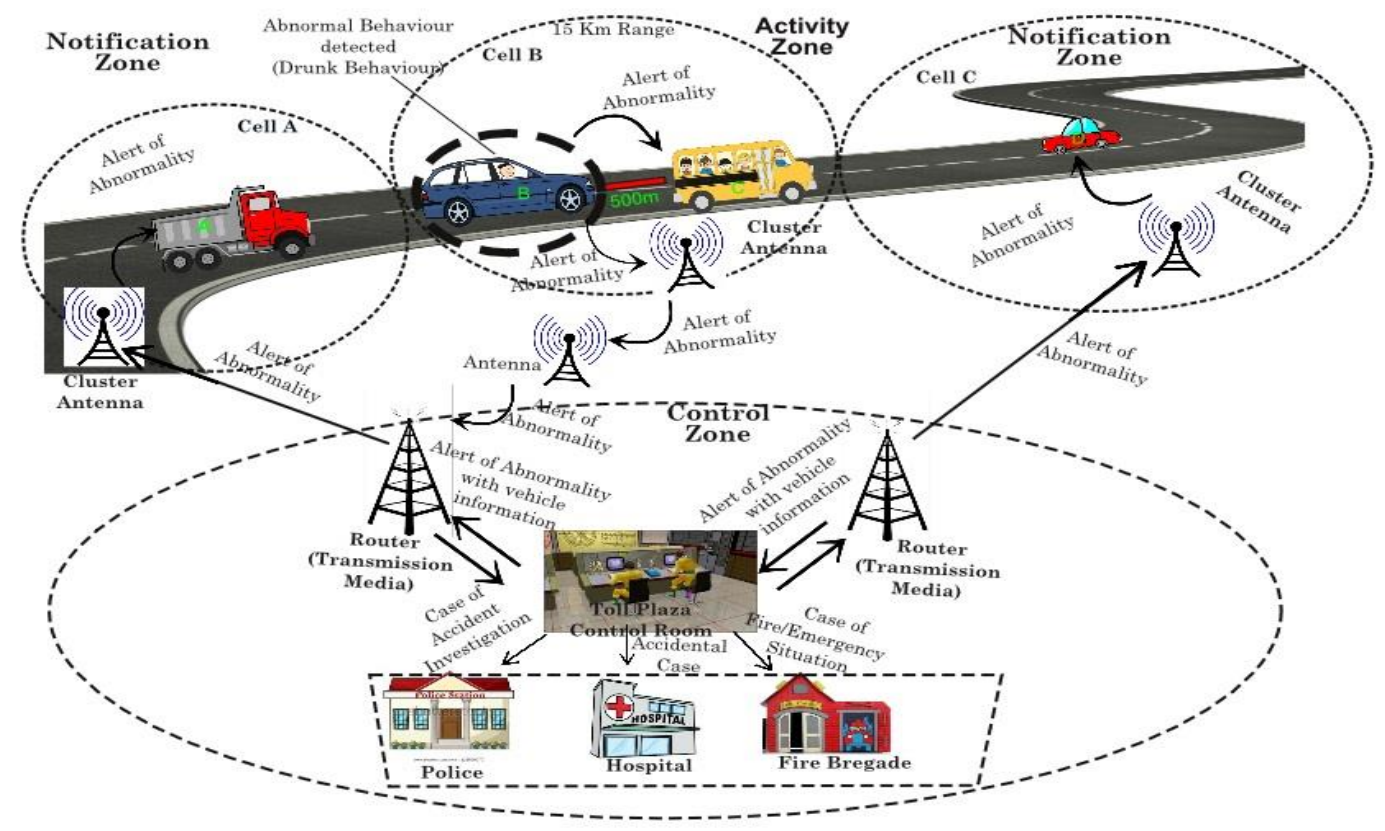

Fig. 6. Pictorial representation of Communication regarding Abnormalities on the highway

Probable Accident condition occurs when accident has not yet happened but there is a possibility of occurrence. In case of probable accident scenario if, abnormal behavior of the vehicle and driver is being detected, the designated sensors of the vehicle will sense the event and alert system will get activated. Intimation can be sent to the vehicles which are in the range of $1 \mathrm{~km}$ from the vehicle in which abnormality is detected. The alert signal can also be sent to cluster antenna of the corresponding cell of the vehicle. Finally the alert will be sent to nearest toll plaza which will act as a con- 
trol room. The control room of the toll plaza can take possible actions like, setting off an alarm within the vehicle, controlling speed of the vehicle etc.

Actual accident condition: occurs when the fatality or accident has occurred. In this case the alert system of the vehicle can get activated and sent alert of the accident to the vehicles surrounding it up to range of $1 \mathrm{~km}$. This alert is intended to make sure that other vehicles don't get trapped in the zone. The information will be sent to nearest toll plaza which is acting as control room. The toll plaza will sent immediate notification to the concerned casualty department like, hospital, police and fire brigade to reach to accident site.

Notification zone: In notification zone, after detecting the abnormality in activity zone the cluster antenna of that zone will receive the information and shower that information to the cluster antenna's of the nearby cells, which will further transmit it to the other vehicles and nearest toll plaza.

Control Zone: In control zone, control room plays an important role, it receives data from nearby cluster antenna, analyse it and can initialize an alert action such as intimating fire brigade in case of fire, hospital in case of trauma care and police.

\section{Conclusion}

In this paper we have proposed an architecture based on wireless sensor network, that can help in monitoring various aspects like vehicle condition, driver behavior in real time if this will be implemented in future can be helpful and effective in reducing the occurrences of accidents up to great extent.

\section{References}

1. A. Mounika, D. Srikar and P.Rajendra Chaitanya "Driver Behaviour Monitoring and Alerting System for Safer Navigation" International Journal Of Research In Advanced Engineering Technologies, Volume 4, Issue 4 Aug (2015)

2. Adnan K. Shaout and Adam E. Bodenmiller "A Mobile Application for Monitoring Inefficient and Unsafe Driving Behaviour"The Electrical and Computer Engineering Department, The University of Michigan-Dearborn Dearborn, Michigan 48128, 2011-12-11

3. D. Sowmya, I.Suneetha and N.Pushpalatha "Driver Behaviour Monitoring Through Sensors And Tracking The Accident Using Wireless Technology" International Journal Of Computer Applications, Vol. 102-No.2, September (2014).

4. JoelC.McCall and Mohan M. Trivedi "Visual Context Capture and Analysis for Driver Attention Monitoring" Computer Vision and Robotics Research Laboratory University of California, San Diego, 7th IEEE Conference on Intelligent Transportation Systems

5. Luis M. Bergasa, Jesus Nuevo, Miguel A. Sotelo and Manuel Vhzquez "Real-Time System For Monitoring Driver's Vigilance" IEEE Transaction of intelligent Transportation Systems, Vol. 7, IEEE Transactions ..., 2006 - ieeexplore.ieee.org

6. Mohamad-HoseynSigari, Mahmood Fathy and Mohsen Soryani "A Review on Driver Face Monitoring Systems for Fatigue and Distraction Detection" International Journal of Advanced Science and Technology Vol.64(2014) 
7. I.G Daza, N. Hernandez, L. M. Bergasa, I. Parra, J. J. Yebes, M. Gavilan, R. Quintero, D. F. Llorca and M. A. Sotelo"Drowsiness Monitoring Based on Driver and Driving Data Fusion" Department of Electronics, University of Alcala Madrid Spain, 2011, 14th International IEEE Conference on Intelligent Transportation Systems, Washington DC, USA.

8. Gys Albertus Marthinus Meiring and Hermanus Carel Myburgh "A Review of Intelligent Driving Style Analysis Systems and Related Artificial Intelligence Algorithms" Received: 14 July (2015); Accepted: 16 November (2015); Published: 4 December 2015, Academic Editor: Felipe Jimenez Department of Electrical, Electronic and Computer Engineering

9. J.Wu, M. McDonald and K. Chatterjee "A Detailed Evaluation of Ramp Metering Impacts on Driver Behavior" Transportation Research Part F 10 (2007) 61-75, Elsevier Ltd.

10. Kelvin C. Baldwin, Donald D. Duncan and Sheila K. West "The Driver Monitor System: A Means Of Assessing Driver Performance" Johns Hopkins Technical Digest, Volume 25, Number 3

11. K.Navya and Dr. M.B.R Murthy "A Zigbee Based Patient Health Monitoring System” Int. Journal of Engineering Research and Applications www.ijera.com Vol. 3, Issue 5, Sep-Oct 2013, pp.483-486

12. Kazem Sohraby, DaniealMinoli and TaiebZnati“ Wireless Sensor Networks- Technology, Protocols, and applications", 2nd Edition by Willey India

13.Prashant Roy, Ankit Khetrapal and Sandeep Rathore "Driver Assistance System" School Of Electronic Engineering, VIT University, Vellore Tamilnadu, India, Volume 2, Issue 4, ISSN:2321-9939

14. QiangJi and Xiaoji Yang "Real-Time Eye, Gaze, and Face Pose Tracking For Monitoring Driver's Vigilance"(2002) Elsevier Science Ltd., Real time imaging 8,357-377(2002)

15.Singh Himani Parmar, Mehul Jajal and Yadav Priyanka Brijbhan "Drowsy Driver Warning System Using Image Processing" INTERNATIONAL JOURNAL OF ENGINEERING DEVELOPMENT AND RESEARCH IJEDR,ISSN: 2321-9939

16.T. Brandt, R. Stemmer, B. Mertsching and A. Rakotonirainy "Affordable Visual Driver Monitoring System for Fatigue and Monotony” 0-7803-8566-7/04/2004 IEEE.

\section{Web References}

17. http://ijtir.hctl.org/vol14/IJTIR_Article_201504011.pdf

18. http://pibphoto.nic.in/documents/rlink/2016/jun/p20166905.pdf, Road Accidents in India 2015

19. http://revista.dgt.es/images/informe-accidentes-India-2013.pdf,Road Accidents in India2013. 